\title{
Retinoic acid receptor $\gamma$ activation promotes differentiation of human induced pluripotent stem cells into esophageal epithelium
}

\author{
Yasufumi Koterazawa ${ }^{1,2,3} \cdot$ Michiyo Koyanagi-Aoi ${ }^{1,2,4} \cdot$ Keiichiro Uehara $^{1,2,5}$. \\ Yoshihiro Kakeji ${ }^{3}$ Takashi Aoi ${ }^{1,2,4}$
}

Received: 17 December 2019/ Accepted: 2 June 2020/Published online: 16 June 2020

(C) The Author(s) 2020

\begin{abstract}
Background The esophagus is known to be derived from the foregut. However, the mechanisms regulating this process remain unclear. In particular, the details of the human esophagus itself have been poorly researched. In this decade, studies using human induced pluripotent stem cells (hiPSCs) have proven powerful tools for clarifying the developmental biology of various human organs. Several studies using hiPSCs have demonstrated that retinoic acid (RA) signaling promotes the differentiation of foregut into tissues such as lung and pancreas. However, the effect of RA signaling on the differentiation of foregut into esophagus remains unclear.

Methods We established a novel stepwise protocol with transwell culture and an air-liquid interface system for esophageal epithelial cell (EEC) differentiation from hiPSCs. We then evaluated the effect of all-trans retinoic
\end{abstract}

Electronic supplementary material The online version of this article (https://doi.org/10.1007/s00535-020-01695-7) contains supplementary material, which is available to authorized users.

Takashi Aoi

takaaoi@med.kobe-u.ac.jp

1 Division of Advanced Medical Science, Graduate School of Science, Technology and Innovation, Kobe University, 7-5-1 Kusunoki-cho, Chuo-ku, Kobe 650-0017, Japan

2 Department of iPS Cell Applications, Graduate School of Medicine, Kobe University, Kobe, Japan

3 Division of Gastrointestinal Surgery, Department of Surgery, Graduate School of Medicine, Kobe University, Kobe, Japan

4 Center for Human Resource Development for Regenerative Medicine, Kobe University Hospital, Kobe, Japan

5 Division of Pathology, Graduate School of Medicine, Kobe University, Kobe, Japan acid (ATRA), which is a retinoic acid receptor (RAR) $\alpha$, $\operatorname{RAR} \beta$ and $\operatorname{RAR} \gamma$ agonist, on the differentiation from the hiPSC-derived foregut. Finally, to identify which RAR subtype was involved in the differentiation, we used synthetic agonists and antagonists of RAR $\alpha$ and $\operatorname{RAR} \gamma$, which are known to be expressed in esophagus.

Results We successfully generated stratified layers of cells expressing EEC marker genes that were positive for lugol staining. The enhancing effect of ATRA on EEC differentiation was clearly demonstrated with quantitative reverse transcription polymerase chain reaction, immunohistology, lugol-staining and RNA sequencing analyses. RAR $\gamma$ agonist and antagonist enhanced and suppressed EEC differentiation, respectively. RAR $\alpha$ agonist had no effect on the differentiation.

Conclusion We revealed that $\operatorname{RAR} \gamma$ activation promotes the differentiation of hiPSCs-derived foregut into EECs.

Keywords Induced pluripotent stem cells - Esophageal epithelial cell differentiation - Retinoic acid signal · Retinoic acid receptor $\gamma$

$\begin{array}{ll}\text { Abbreviations } \\ \text { iPSCs } & \text { Induced pluripotent stem cells } \\ \text { RA } & \text { Retinoic acid } \\ \text { EEC } & \text { Esophageal epithelial cell } \\ \text { ATRA } & \text { All-trans retinoic acid } \\ \text { RAR } & \text { Retinoic acid receptor } \\ \text { RT- } & \text { Reverse transcription polymerase chain reaction } \\ \text { PCR } & \\ \text { PBMCs } & \text { Peripheral blood mononuclear cells } \\ \text { DE } & \text { Definitive endoderm } \\ \text { FG } & \text { Foregut } \\ \text { dAFG } & \text { Dorsal anterior foregut } \\ \text { ALI } & \text { Air-liquid interface }\end{array}$


TPM Transcripts per million

FPKM Fragments per kilobase of exon per million mapped fragments

PFG Posterior FG

vAFG Ventral anterior foregut

\section{Introduction}

The esophagus is known to be derived from the anterior region of the foregut via tracheal-esophageal separation in embryogenesis. Previous studies using mouse models have identified several important signals in the development of esophageal epithelium [1, 2]. However, the molecular mechanisms regulating this process remain to be fully clarified. In particular, relatively little information is available concerning the developmental process of human esophageal epithelial cells (EECs).

Human tissue organoids derived from human pluripotent stem cells have proven powerful tools for research on the developmental biology of various human organs [3]. For example, several reports using *human-induced pluripotent stem cells (hiPSCs) have identified signals and transcriptional factors that play essential roles in the development of foregut-derived organs, such as the liver, lung and stomach [4-6]. However, there have been only two reports concerning the generation of human esophagus from iPSCs [7, 8], and neither resolved molecular mechanisms underlying the differentiation protocols employed.

All-trans retinoic acid, which binds to $\operatorname{RAR} \alpha, \operatorname{RAR} \beta$ and RAR $\gamma$ as a pan RAR agonist, has shown to promote differentiation of various endodermal tissues, including lung, pancreas and bladder $[5,9,10]$, and tissues consisting of squamous cells, such as skin and cornea [11, 12], in several studies using human cells. In mouse models, retinoid acid signaling enhanced the terminal differentiation and proliferation of esophageal progenitor cells [13, 14], but the effects of retinoid acid signaling on the earlier developmental processes of esophagus have never been reported. Regarding the process of differentiation from human foregut into esophageal epithelium, the effect of retinoic acid signaling remains unclear.

In this study, we generated human esophageal epithelium from hiPSCs using a novel stepwise differentiation protocol and evaluated the effect of retinoic acid signaling on the processes. We found that RAR $\gamma$ activation promotes the differentiation of human anterior foregut into EECs.

\section{Methods}

\section{iPSC culture}

We used the iPSC line FF-PB-3AB4, which was established from a healthy donor's peripheral blood mononuclear cells (PBMCs) [15]. The institutional review board of the Kobe University Graduate School of Medicine approved this study (No. 1722), and informed consent was obtained from the donor.

We cultured the iPSC line FF-PB-3AB4 according to a previously described method [16]. In brief, the iPSCs were cultured with iMatrix-511 silk $\left(0.25 \mu \mathrm{g} / \mathrm{cm}^{2}\right.$; Nippi, Tokyo, Japan) in uncoated usage and maintained in StemFit medium (Ajinomoto, Tokyo, Japan) with $50 \mathrm{U} / \mathrm{ml}$ of penicillin and $50 \mu \mathrm{g} / \mathrm{ml}$ of streptomycin (Life Technologies, Waltham, MA, USA) at $37{ }^{\circ} \mathrm{C}$ with $5 \% \mathrm{CO}_{2}$. The medium was changed every other day. The cells were passaged every 5-8 days using $0.5 \times$ TrypLE Select $(1 \times$ TrypLE Select diluted 1:1 with $0.5 \mathrm{mM}$ EDTA/PBS [-]; Life Technologies) and cultured in StemFit medium supplemented with $10 \mu \mathrm{M}$ of Rock inhibitor (Y-27632; WAKO, Osaka, Japan) for 1 day.

\section{EEC differentiation}

For differentiation into definitive endoderm (DE), $1.8 \times 10^{5}$ iPSCs were seeded as single cells on 24-well plates pre-coated with iMatrix-511 silk $\left(0.5 \mu \mathrm{g} / \mathrm{cm}^{2}\right)$ in StemFit medium supplemented with $10 \mu \mathrm{M}$ of ROCK inhibitor Y-27632 (WAKO). The next day, the medium was changed to RPMI 1640 medium (Nacalai Tesque, Kyoto, Japan) supplemented with $100 \mathrm{ng} / \mathrm{ml}$ of Activin A (Peprotech, Rocky Hill, NJ, USA), 1 or $3 \mu \mathrm{M}$ of CHIR99021 (TOCRIS, Bristol, UK), 2\% B27 (Life Technologies), $2 \mathrm{mM} L$-glutamine (Life Technologies), penicillin and streptomycin, and cells were cultured until Day 3.

To generate foregut (FG), cells were cultured with RPMI 1640 medium supplemented with $2 \%$ fetal bovine serum (FBS), $2 \mu \mathrm{M}$ of CHIR99021 (TOCRIS), $100 \mathrm{ng} / \mathrm{ml}$ of LDN193189 (Cellagen Technology, San Diego, CA, USA), penicillin and streptomycin from Day 3 to Day 6 . SB431542 (5 $\mu \mathrm{M}$; WAKO) was added on Day 4 and Day 5.

For induction of dorsal anterior foregut (dAFG), cells were cultured with RPMI 1640 medium supplemented with $2 \%$ FBS, $2 \mu \mathrm{M}$ of CHIR99021 (TOCRIS), $0.5 \mu \mathrm{M}$ ATRA (WAKO), $10 \mathrm{ng} / \mathrm{ml}$ of FGF10 (WAKO), $10 \mathrm{ng} / \mathrm{ml}$ of KGF (WAKO), $10 \mathrm{ng} / \mathrm{ml}$ of BMP4 (R\&D Systems, Minneapolis, MN, USA), $10 \mathrm{ng} / \mathrm{ml}$ of EGF (R\&D Systems), penicillin and streptomycin from Day 6 to Day 13 . 
For terminal differentiation into EECs, cells were cultured with RPMI 1640 medium supplemented with KGMGold SingleQuots (Sup-KGM), containing 0.1\% hydrocortisone, $0.1 \%$ transferrin, $0.05 \%$ epinephrine $0.25 \mathrm{ml} / \mathrm{L}$, $0.1 \%$ GA-1000, $0.4 \%$ bovine pituitary extract, $0.1 \%$ human epidermal growth factor, insulin $0.5 \mathrm{ml} / \mathrm{L}$ (LONZA, Basel, Switzerland), $10 \mathrm{ng} / \mathrm{ml}$ of BMP4, $0.5 \mu \mathrm{M}$ ATRA, $10 \mathrm{ng} / \mathrm{ml}$ of EGF, penicillin and streptomycin from Day 13 to Day 21 (Figs. 1, 3, 4) or Day 24 (Figs. 2, 5). Sup-KGM is a supplement for keratinocyte basal medium (Lonza) and used for differentiation into bladder urothelial cells, which also have stratified structures [15].

\section{$\mathrm{RA}$ receptor-specific agonists and antagonists}

To assesses which RA receptor subtype plays an essential role in esophageal differentiation, $1 \mu \mathrm{M}$ of the RAR $\alpha$ agonist AM580 (Cayman Chemical, Ann Arbor, MI, USD), $2 \mu \mathrm{M}$ of the RAR $\beta$ agonist CD2314 (R\&D Systems) and $0.5 \mu \mathrm{M}$ of the RAR $\gamma$ agonist BMS961 (R\&D Systems) were added from Day 6 to Day 21 (Fig. 4) or Day 24
Fig. 1 Induction of EECs from hiPSCs. a A schematic diagram of the experiment to examine the conditions underlying the differentiation of foregut (FG) into dorsal anterior foregut (dAFG). b An expression analysis of SOX2 and p63 at Day 13 by semi-quantitative RT-PCR. GAPDH was used as an endogenous control. c Immunostaining of SOX2 (green), p63 (red) and Hoechst (blue) at Day 13. Representative fluorescence is shown. Scale bars, $50 \mu \mathrm{m}$. d A schematic diagram of the experiment to examine the conditions underlying the differentiation of dAFG into EECs. e Expression analyses of p63, SOX2, CK13, CK5 and PAX9 at Day 21 by semi-quantitative RT-PCR. GAPDH was used as an endogenous control. Total RNA of human normal esophageal tissue was used as a positive control
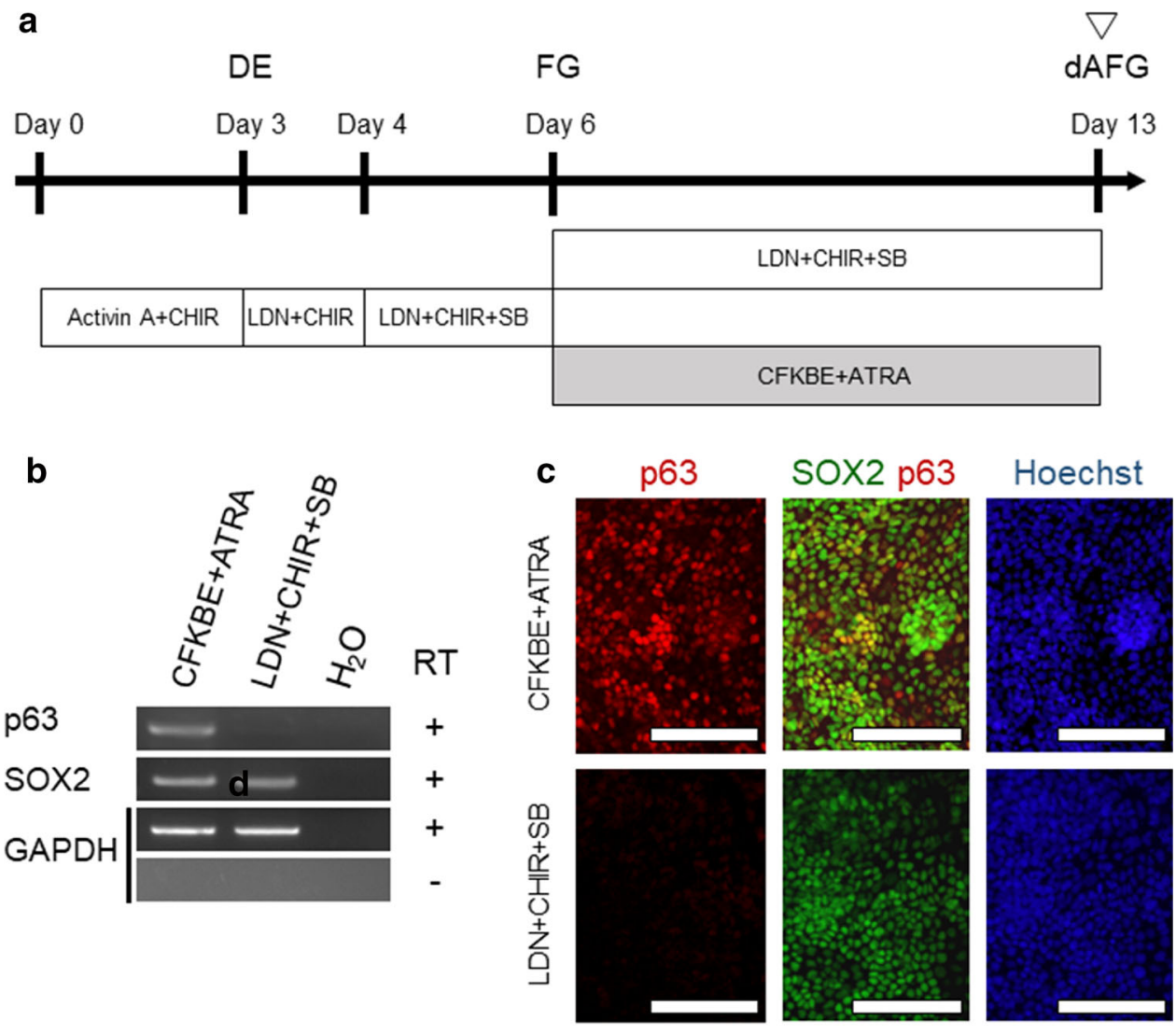

d

e

$50 \mu \mathrm{m}$

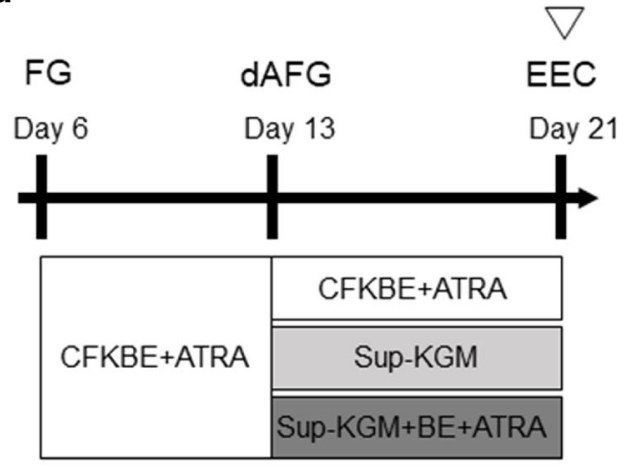

p63
SOX2
CK13
CK5
PAX9

GAPDH 
Fig. 2 The expression of esophageal epithelial marker proteins in the hiPSC-derived cells and fetal mouse esophagus at E17.5. a A schematic representation of the stepwise differentiation protocol and marker genes at each step. b Immunostaining of the SOX2, p63, CK5, E-cadherin, CK13 and PAX9 at Day 24 in the derivatives of hiPSCs (upper panels) and fetal mouse esophagus at E17.5 (lower panels). Scale bars, $20 \mu \mathrm{m}$ a

$\begin{array}{ll} & \text { LDN } \\ \text { Activin A } & \text { CHIR } \\ \text { CHIR } & \text { SB }\end{array}$

CHIR, ATRA

FGF10, KGF

Sup-KGM, BMP4,

SB

BMP4, EGF

ATRA, EGF

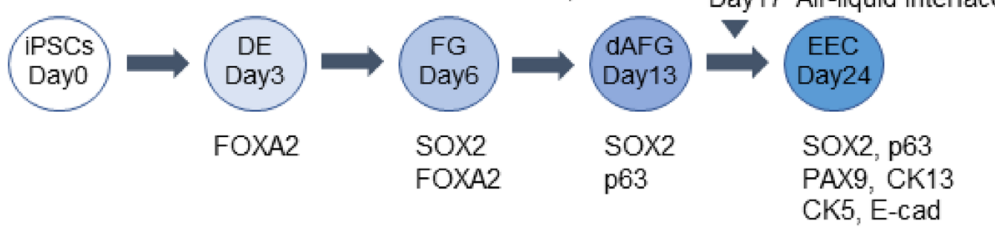

b

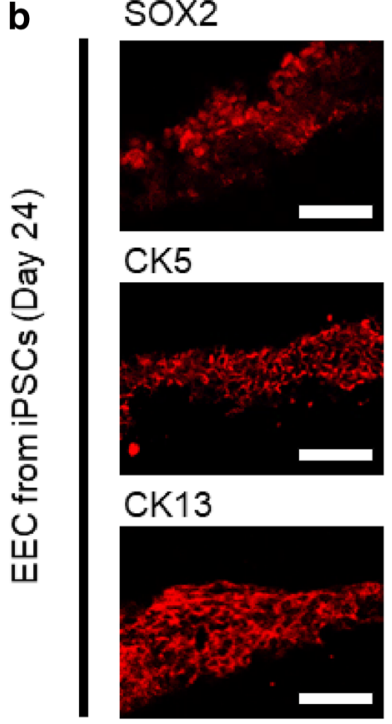

p63

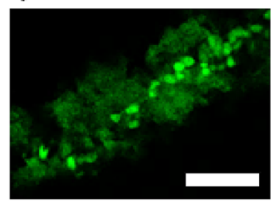

Hoechst

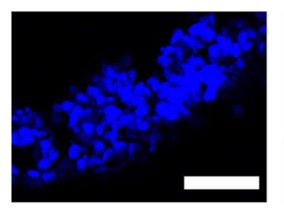

Merge

\section{E-cadherin}

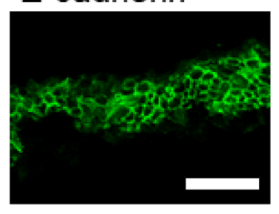

Hoechst
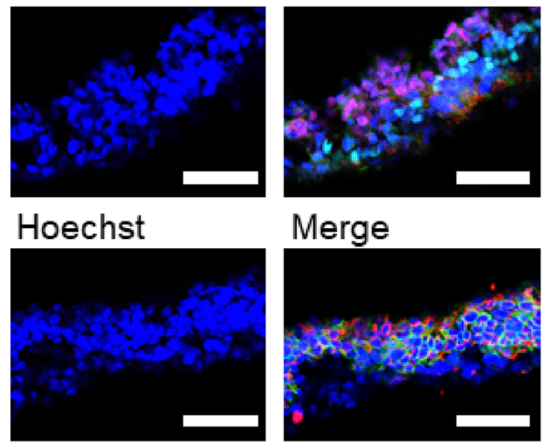

Merge

PAX9

Hoechst

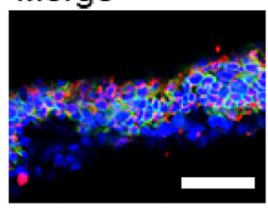

Merge
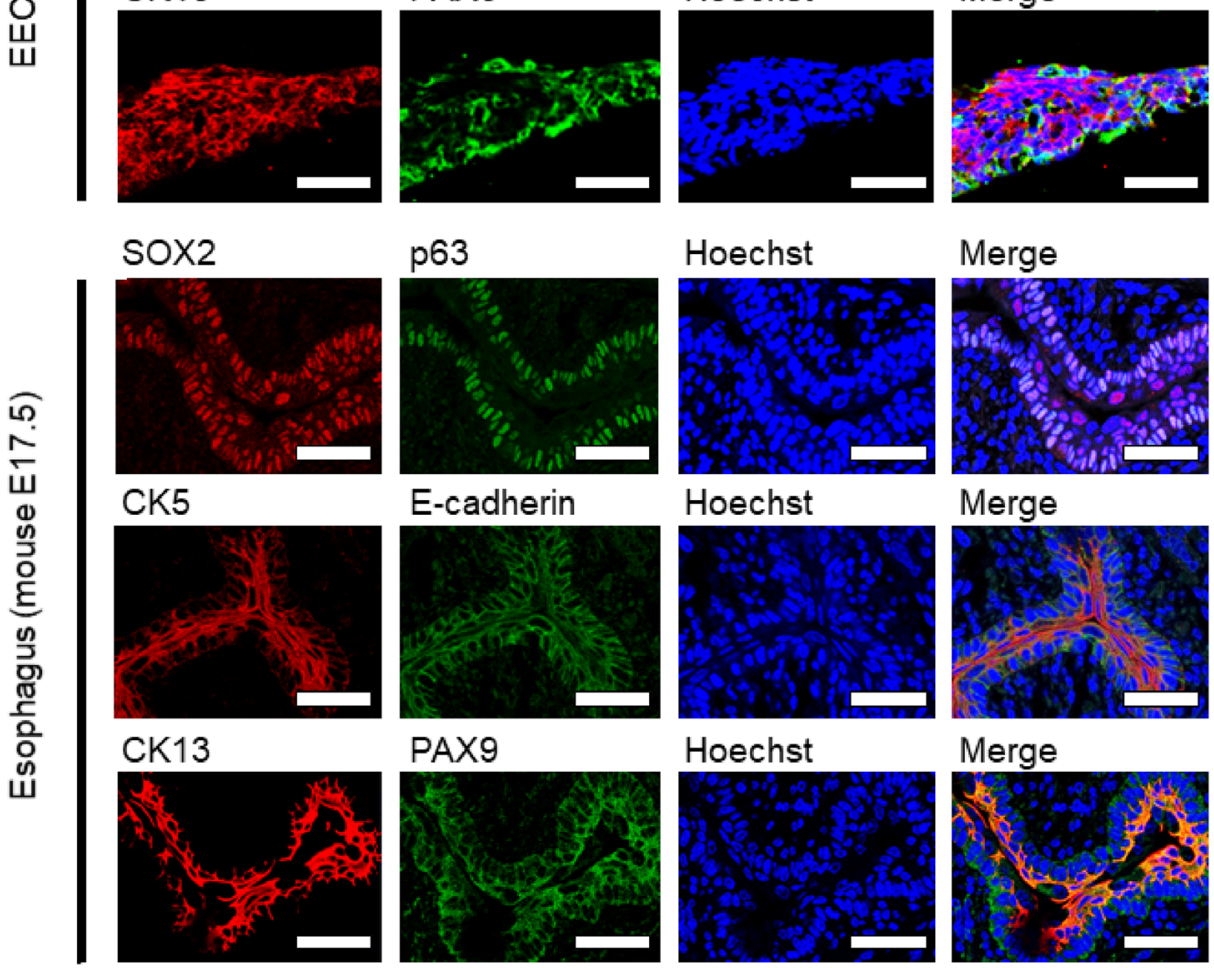

p63

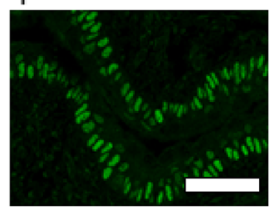

Hoechst

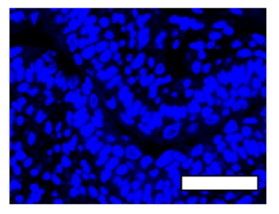

Merge

E-cadherin

Hoechst

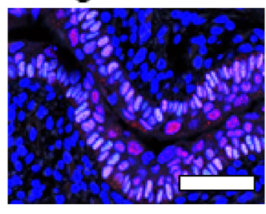

Merge
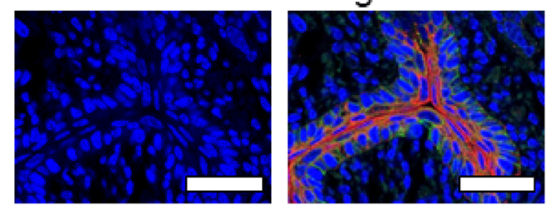

Hoechst
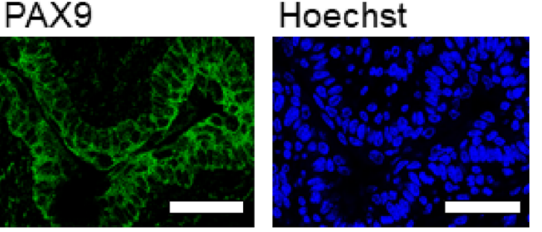

Merge

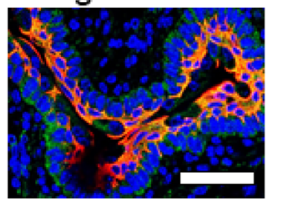

$\square 20 \mu \mathrm{m}$

(Fig. 5). In addition, $0.5 \mu \mathrm{M}$ of ATRA with $2 \mu \mathrm{M}$ of the RAR $\gamma$ antagonist MM11253 (R\&D Systems) or $4 \mu \mathrm{M}$ of the RAR $\gamma$ antagonist LY2955303 (R\&D Systems) were added from Day 6 to Day 21.
Transwell culture with air-liquid interface (ALI)

For the in vitro $3 \mathrm{D}$ reconstruction, $1.8 \times 10^{5}$ iPSCs were seeded onto transwells $(0.4-\mu \mathrm{m}$ pore size; Corning, Corning, NY, USA) pre-coated with iMatrix-511 silk $(0.5 \mu \mathrm{g} /$ $\mathrm{cm}^{2}$ ). The protocol of differentiation was the same as that of normal culture. The ALI procedure was started on Day 
Fig. 3 Enhancing effects of ATRA on the esophageal epithelial differentiation. a A schematic diagram of the experiment to examine the effects of ATRA on the differentiation of foregut cells into esophageal epithelium. b Lugol staining at Day 21. c Immunostaining of CK13 (red) at Day 21 (left panels). The right panels show merged images of CK13 and Hoechst (blue) staining. Scale bars, $20 \mu \mathrm{m}$. (d, e) Transcriptome data analyses of the cells derived with or without ATRA at Day 21. The scatterplot shows the expression of esophagealspecific genes (red dots), retinoid target genes (green dots) and other genes (gray dots) (d). The heat map shows the average Z-scores of esophageal-specific genes and retinoid target genes (e). f RTPCR analyses of the lung marker genes SFTPB, SFTPC and NKX 2.1 at Day 21. GAPDH was used as an endogenous control. Total RNA of human normal lung tissue was used as a positive control. (g) RT-PCR analyses of stomach marker genes: TFF1, TFF2 and MUC5AC. GAPDH was used as an endogenous control at Day 21. Total RNA of human normal stomach tissue was used as a positive control a

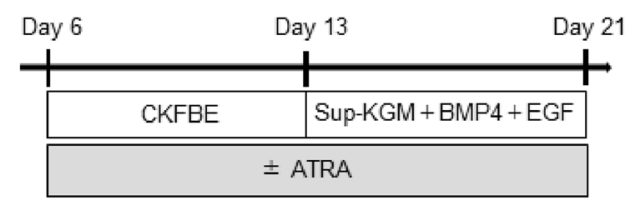

b

ATRA -
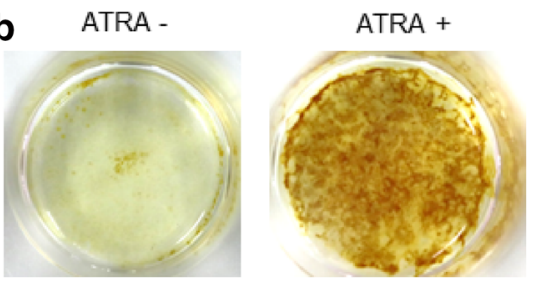

C
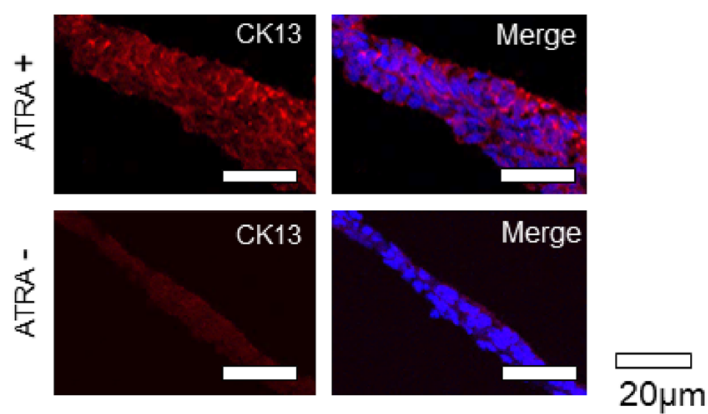

d

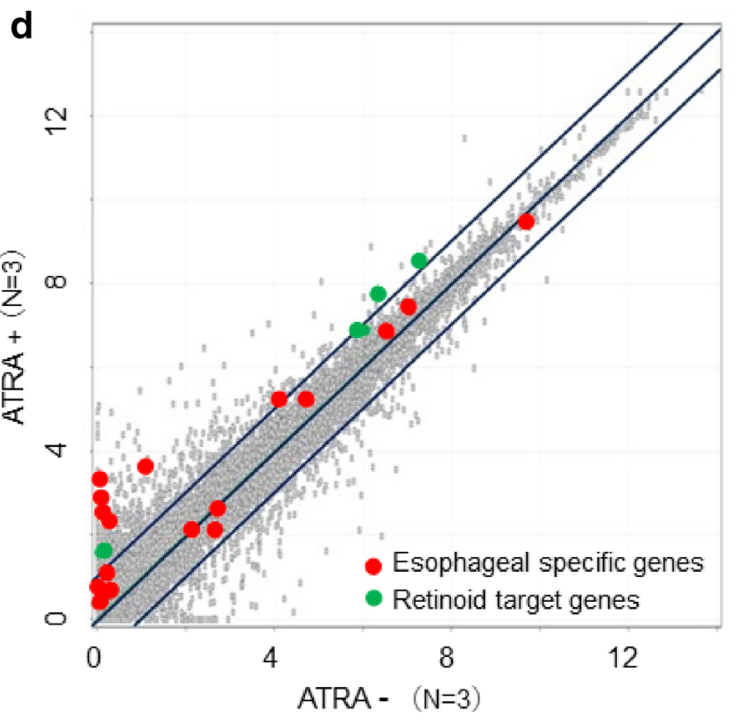

e

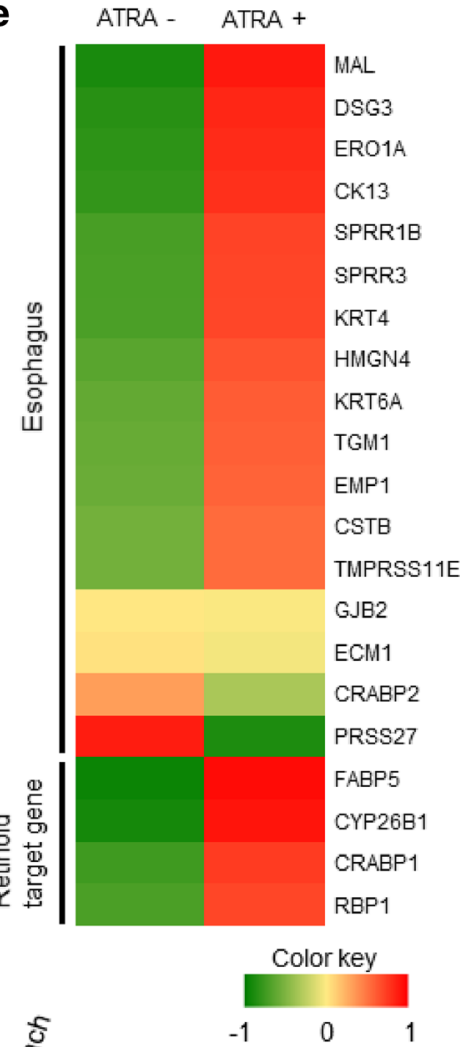

$\mathbf{f}$

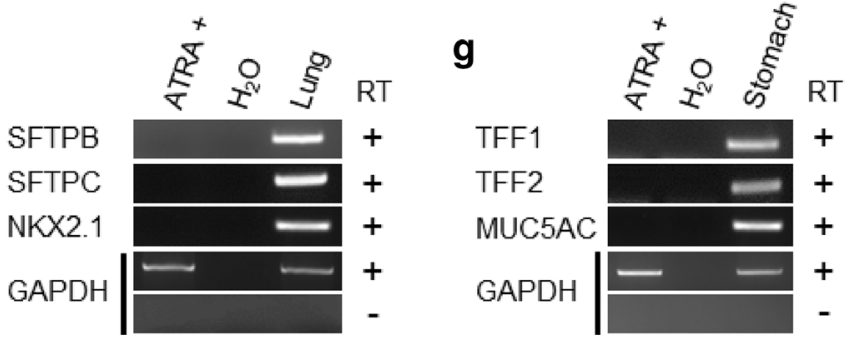

17, and cells were exposed to the ALI by removal of the medium in the upper compartment until Day 24 [17].

\section{Semi-quantitative or real-time quantitative reverse transcription polymerase chain reaction (RT-PCR)}

Total RNA was isolated using Trizol (Life Technologies) and treated with the TURBO DNA-free kit (Thermo Fisher Scientific, Waltham, MA, USA). The Prime Script II 1st strand cDNA Synthesis Kit (Takara, Shiga, Japan) was used to synthesize cDNA from 500 to $800 \mathrm{ng}$ of total RNA.

For semi-quantitative RT-PCR (Figs. 1b, e, 3f, g, S-Figs. 2a, 2b, 8a, 8b), the resulting cDNA was subjected to PCR with a Takara Ex Taq PCR kit (Takara). In Figs. 4b, c, e, f and S-Figs 1d, 4b, 6b, 6d, 7b, 7c, a realtime quantitative RT-PCR analysis was performed using TB Green Premix Ex Taq (Takara) on a Light Cycler 480 II (Roche, Basel, Switzerland). The PCR primers used are listed in Supplementary Table 1. 
Fig. 4 ATRA enhances differentiation into EECs via RAR $\gamma$ receptor. a A schematic diagram of the experiment to compare the effects of AM580 (RAR $\alpha$-specific agonist), BMS961 (RAR $\gamma$-specific agonist) and ATRA on EEC differentiation. (b,

c) Expression analyses of FABP5, SOX2, CK13, PAX9 and S100A14 in the differentiated cells treated with AM580 (b) or BMS961 (c) by qRT-PCR. GAPDH was used as an endogenous control. Data represent the mean \pm SEM $(n=6) . * p<0.05$ from an ANOVA with Tukey's test. d A schematic diagram of the experiment to examine the effects of adding LY2955303 (RAR $\gamma$ antagonist) to ATRA treatment on EEC

differentiation. e Expression analyses of FABP5, SOX2,

CK13, PAX9 and S100A14 in differentiated cells treated with LY29953 by qRT-PCR.

GAPDH was used as an endogenous control. Data represent the mean \pm SEM $(n=6) . * p<0.05$ from an ANOVA with Tukey's test. (f) Expression analyses of RAR $\gamma$ in iPSCs (Day 0), FG (Day 6), dAFG (Day 13) and EECs (Day 21). RPL27 was used as an endogenous control. Data represent the mean $\pm \operatorname{SEM}(n=3)$ a

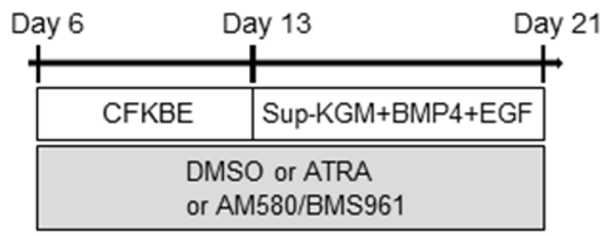

b
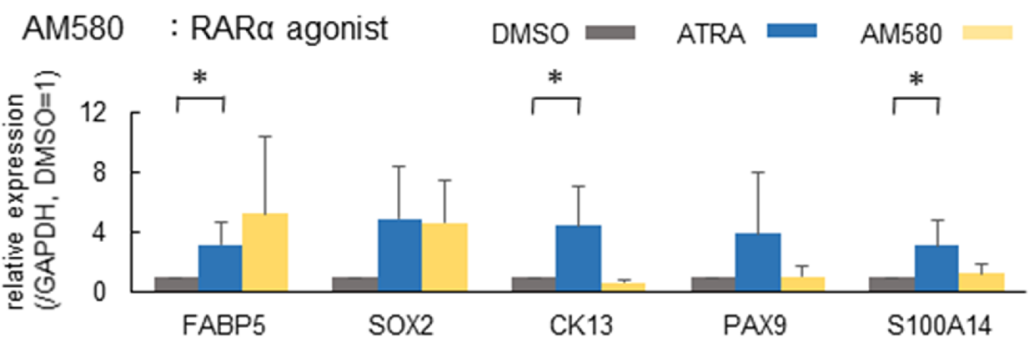

C BMS961 : RARy agonist

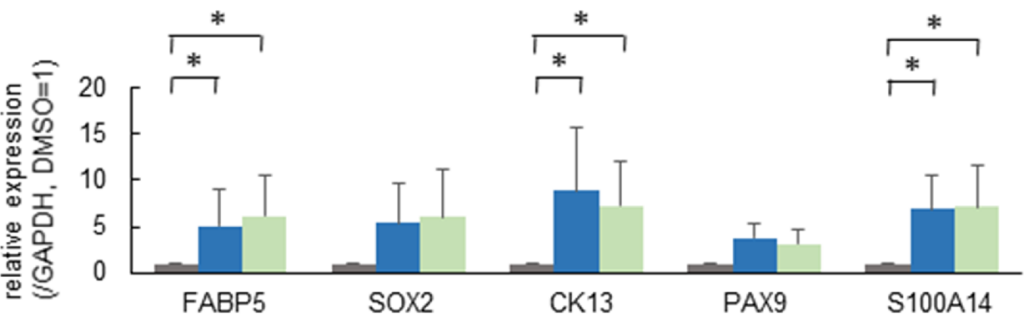

d

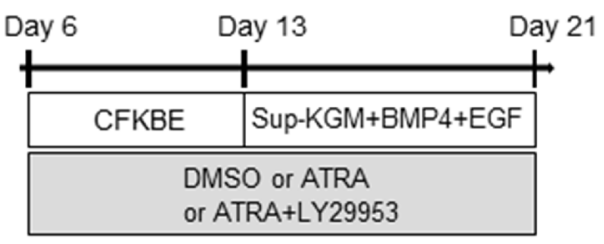

e

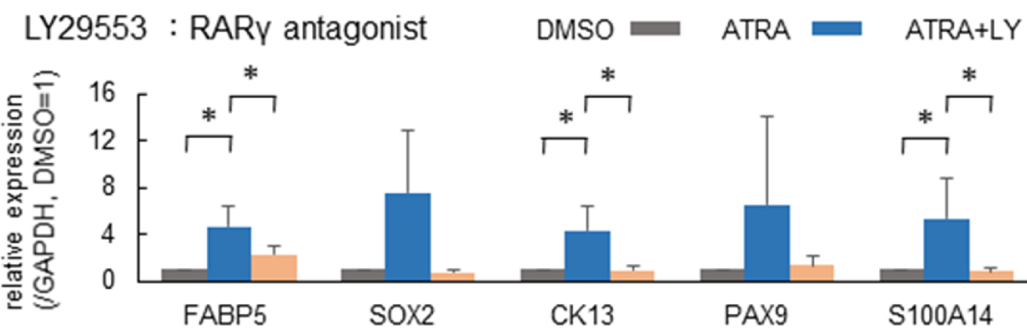

f

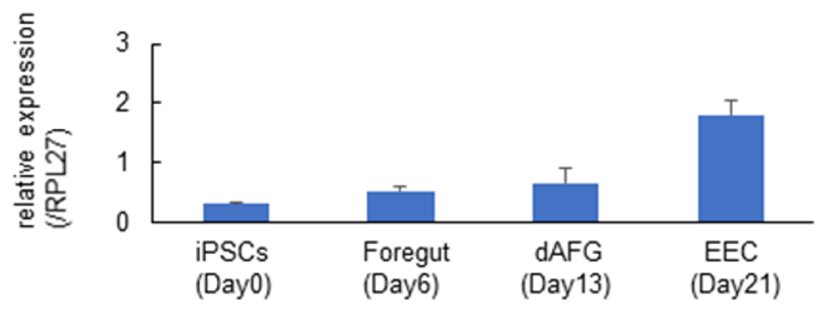

\section{Immunocytochemistry}

Cells were fixed with $10 \%$ formalin for $10 \mathrm{~min}$ at room temperature. After being washed with PBS, the cells were treated with $0.2 \%$ Triton X-100 in PBS and blocked with
5\% donkey serum and $1 \%$ BSA in PBS for 45 min at room temperature. The cells were incubated with primary antibodies at $4{ }^{\circ} \mathrm{C}$ overnight and then stained with secondary antibodies. The primary antibodies were rabbit anti-SOX2 (ab97959, dilution 1:200; abcam, Cambridge, UK) and 
Fig. 5 Treatment with an $\operatorname{RAR} \gamma$-specific agonist instead of ATRA also induced stratified layers of cells expressing esophageal epithelial markers. a A schematic diagram of the EEC differentiation protocol with an RAR $\gamma$-specific agonist. b Immunostaining of SOX2, p63, CK5, E-cadherin, CK13 and PAX9 in the derivatives of hiPSCs. Scale bars, $20 \mu \mathrm{m}$ a

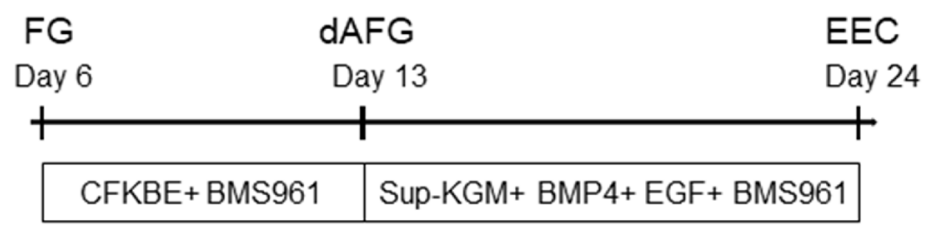

b

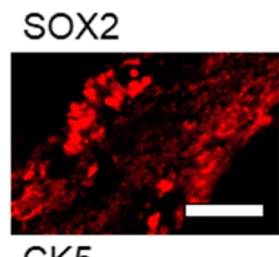

CK5
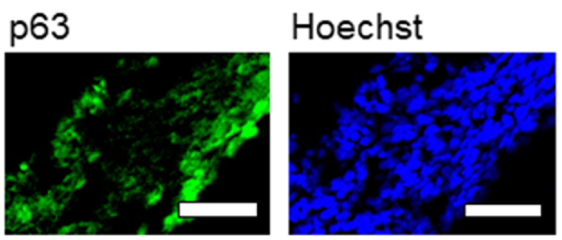

Merge

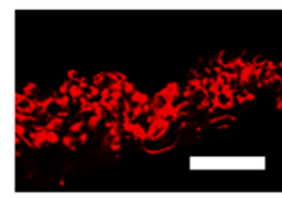

E-cadherin

Hoechst
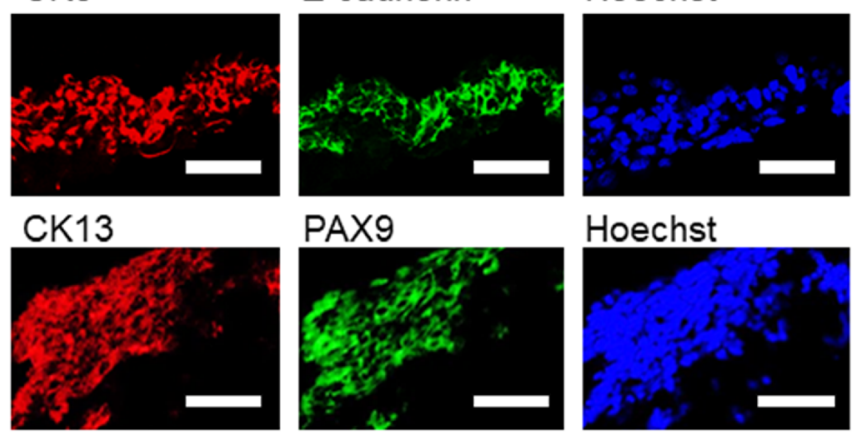

PAX9

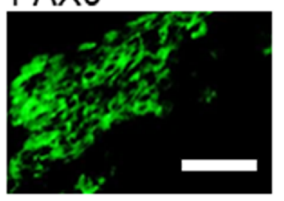

Hoechst

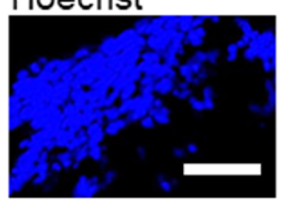

Merge

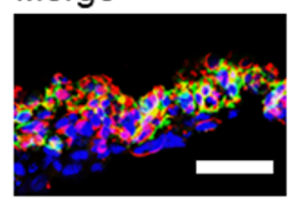

Merge

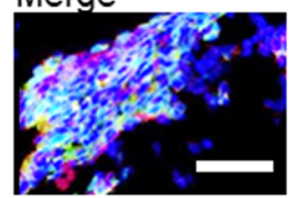

goat anti-p63 (BAF1916, dilution 1:200; R\&D Systems). All of the secondary antibodies (Alexa Fluor 488-conjugated anti-rabbit IgG and Alexa Fluor 594-conjugated antigoat IgG) were obtained from Life Technologies. Hoechst 33,342 (WAKO) was used for nuclear staining.

\section{Frozen section samples}

The cultured organoids were fixed with $10 \%$ formalin and then embedded in Tissue-Tek O.C.T Compound (Sakura Finetek Japan, Tokyo, Japan) and frozen at $-80{ }^{\circ} \mathrm{C}$. The frozen samples were sectioned at $8 \mu \mathrm{m}$ on a cryostat.

\section{Immunohistochemical analyses of the organoids}

For immunohistofluorescence, the sections were treated with $0.2 \%$ Triton X-100 in PBS and blocked with 5\% donkey serum and $1 \% \mathrm{BSA}$ in PBS for $45 \mathrm{~min}$ at room temperature. Primary antibodies were incubated overnight at $4{ }^{\circ} \mathrm{C}$. Slides were washed in PBS and incubated with secondary antibody for $1 \mathrm{~h}$ at room temperature. The primary antibodies were rabbit anti-SOX2 (ab97959; abcam), rabbit anti-CK13 (ab92551, dilution 1:200; abcam), goat anti-p63 (BAF1916, dilution 1:200; R\&D Systems), rat anti-PAX9 (ab28538, dilution 1:200; abcam), goat anti-Ecadherin (AF648, dilution 1:200; R\&D Systems), rabbit
anti-CK5 (GTX113219, dilution 1:200; GeneTex, Irvine, CA, USA), rabbit anti-Claudin4 (ab210796, dilution 1:200; abcam) and rabbit anti-Involucrin (ab53112, dilution 1:200; abcam). All of the secondary antibodies (Alexa Fluor 594-conjugated anti-mouse or anti-goat IgG, Alexa Fluor 488-conjugated anti-mouse, anti-goat, anti-rat or anti-rabbit $\mathrm{IgG}$ ) were obtained from Life Technologies. Hoechst 33342 (WAKO) was used for nuclear staining.

\section{Lugol staining}

After being washed with PBS, the cells were treated with lugol solution $(1.2 \%$ potassium iodine and $0.6 \%$ iodine in water) and then washed again with PBS. Lugol solution was kindly provided by Kobe University Hospital (Kobe, Japan).

\section{RNA sequencing}

Total RNA was isolated using Trizol (Life Technologies) and treated with the TURBO DNA-free kit (Thermo Fisher Scientific), as described above. The RNA was sent to Macrogen (Seoul, South Korea, https://www.macrogen. com) for library preparation and paired-end RNA sequencing on the Illumina Novaseq6000 platform. Raw sequence files (fastq) were aligned to the human 
transcriptome (hg38) reference sequences using the Strand NGS software program (Strand Life Science, Karnataka, India) with default parameters. The aligned reads were normalized using Transcripts per million (TPM) again with the Strand NGS software program. RNA-seq data have been deposited in the Gene Expression Omnibus (GEO) under accession number GSE140767.

\section{Extraction of esophagus-specific genes and $\mathrm{Z}$ score transformation}

We defined tissue-specific markers as those with a $>$ fivefold higher average Fragments per kilobase of exon per Million mapped fragments (FPKM) level in esophagus tissue than in all other tissues [18]. After normalizing with TPM, we performed log base two transformation. The average Z-scores of tissue-specific genes were then calculated using Excel 2016 (Microsoft, Redmond, WA, USA), and a heat map was created using Excel 2016 (Microsoft).

\section{Statistical analyses}

All data were analyzed using the $\mathrm{JMP}^{\circledR} 10$ software program (SAS Institute Inc., Cary, NC, USA). Differences in the mean values between two groups were analyzed using the paired $t$ test, and three groups were analyzed using an analysis of variance Tukey test. The differences were considered to be statistically significant for $P$ values $<0.05(*)$.

\section{Results}

\section{Induction of EECs from hiPSCs}

Based on previously reported $\mathrm{FG}$ endoderm induction protocols [4-6, 10, 19-22], we developed an original stepwise differentiation method from hiPSCs into EECs via DE, FG and dAFG.

First, hiPSCs were induced to differentiate into DE by treatment with the WNT activator CHIR99021 (hereafter CHIR) and Activin A for 3 days. We then differentiated DE into FG with a combination of LDN193189 (hereafter LDN) and SB431542 with CHIR. We confirmed the obvious expression of the FG marker genes SOX2 and FOXA2 by RT-PCR and immunocytochemistry (S-Fig. 1a and $b$ ). Since previous reports have shown that efficient FG induction requires an appropriate concentration of CHIR during DE induction [15, 19],

we examined the expression of the FG marker SOX2 and $\mathrm{mid} /$ hindgut marker CDX2 at Day 6 when cultured with 1 or $3 \mu \mathrm{M}$ of CHIR during DE induction (S-Fig. 1c) [23]. Quantitative PCR showed that $1 \mu \mathrm{M}$ CHIR treatment induced a significantly higher expression of SOX2 and lower expression of CDX2 than $3 \mu \mathrm{M}$ CHIR treatment (SFig. 1d). Based on these results, we used $1 \mu \mathrm{M}$ of CHIR for DE induction in our protocol.

Next, to induce differentiation of FG into dAFG, we tested two conditions: (1) LDN, CHIR and SB431542 $(\mathrm{LDN}+\mathrm{CHIR}+\mathrm{SB})$ or (2) CHIR, FGF10, KGF, BMP4, EGF and ATRA(CFKBE + ATRA) [20] (Fig. 1a). At Day $13,(2)$ resulted in an enhanced expression of p63, which is a marker for dAFG (esophageal progenitor) and is expressed in basal cells of the esophageal epithelium [2, 24], at the mRNA level (Fig. 1b) and protein level (Fig. 1c). In contrast, NKX2.1, a marker for ventral AFG (lung progenitor) and GATA4, a marker for posterior FG (gastric progenitor), were not expressed at this point (SFig. $2 \mathrm{a}$ and $2 \mathrm{~b}$, respectively).

To induce differentiation of dAFG cells into esophageal lineages, we tested three conditions after Day 13 (Fig. 1d). The first condition involved continued culture under the same conditions as before Day 13 (CFKBE + ATRA). Under the second condition, we used Sup-KGM (see the "Materials and Methods "section), as we previously succeeded in generating bladder urothelium, which shares stratified epithelial structures with esophageal epithelium, using Sup-KGM [15]. Under the third condition, we omitted lung specification reagents (CHIR, FGF10 and KGF) [20] from the CFKBE + ATRA cocktail and added Sup-KGM. After 8 days' culture, the expression of esophagus marker genes (SOX2, p63 and PAX9) were detected under all three conditions. However, the expression of the mature esophageal epithelial markers CK5 and CK13 [21] was detected only in the cells generated under the third condition (Sup-KGM + BE + ATRA) (Fig. 1e).

\section{Expression of esophageal marker genes in the differentiated cells with ALI culture}

A stratified structure is an important property of esophageal epithelium. We, therefore, employed ALI culture on a membrane (transwell culture system) as ALI culture enable us to assess vertical sections of the epithelium $[25,26]$ and has been reported to induce stratified structure formation of primary EEC culture [27]. Our differentiation protocol and the marker genes at each step are shown in Fig. 2a. On Day 24, we performed immunohistochemistry for the derivatives. We deduced that, like derivatives of hiPSCs in previous studies [7, 8], the derivatives in the present study would exhibit fetal histology but not adult histology, which would probably require more long-term differentiation culture. We, therefore, used esophagus tissues of fetal mice (E17.5) as positive controls, as we were unable to obtain human fetal esophagus, which was the preferable positive control. Mouse esophagus is known to have 
keratinized layers, while human esophageal epithelium is not keratinized, and cells retain their nucleus [21]. Our HE staining findings confirmed this species difference in keratinization (S-Fig.3).

All of the esophageal marker genes we tested (SOX2, p63, CK5, E-cadherin, CK13, PAX9, Claudin-4 and Involucrin) were obviously expressed in the differentiated cells from hiPSCs (Fig. 2b and S-Fig. 3, upper panels), similar to the esophagus of mice (E17.5) (Fig. 2b and S-Fig. 3, lower panels). The findings of HE staining (SFig. 3) and the obvious expression of cell-cell adhesion molecules (E-cadherin and Claudin-4) suggested that the cells differentiated with our protocol formed stratified EEC layers but not piled-up collective cells without cell-cell adhesion. Notably, CK13 is expressed in the suprabasal and apical region but not in the basal region in authentic esophageal epithelium [8, 21]; all layers were positive for CK13 in our hiPSC-derived tissues, indicating that the tissues did not identically recapitulate an authentic human esophageal epithelium structure.

\section{Enhancing effects of ATRA on esophageal epithelial differentiation}

We next evaluated the effects of ATRA in our esophageal differentiation protocol (Fig. 3a), as the effects of ATRA in the differentiation of FG have not been clarified [8, 10, 20]. First, we differentiated the cells into an esophageal lineage with $0,0.1,0.5$ and $1.0 \mu \mathrm{M}$ of ATRA from Day 6 (FG) to Day 21 (EEC) (S-Fig. 4a) to identify the appropriate concentration of ATRA for this study. Quantitative PCR showed that 0.5 and $1.0 \mu \mathrm{M}$ ATRA treatment induced a higher expression of SOX2, CK13 and FABP5 than other concentrations (S-Fig. 4b). Considering the toxicity, the lower concentration of $0.5 \mu \mathrm{M}$ was used for the subsequent experiments. The differentiation protocol devoid of ATRA obviously resulted in reduced staining of glycogen with lugol staining (Fig. 3b) and CK13 according to immunohistology (Fig. 3c), suggesting that ATRA plays a critical role in differentiation into esophagus.

We then analyzed the transcriptome data of the cells derived with or without ATRA. The scatterplot (Fig. 3d) and Z-score heatmap (Fig. 3e) showed the upregulation of 13 of the 17 esophagus-specific genes that were extracted from publicly available RNA-seq data (see the "Material and Methods" section) as well as known retinoid target genes [28] in the ATRA $(+)$ cells.

Furthermore, since RA signaling is reported to have positive effects on lung [20] and posterior FG differentiation [8], we examined the expression of marker genes of lung (SFTEB, SFTPC and NKX2.1) and stomach, which is the posterior FG derivative, (TFF1, TFF2 and MUC5AC) in $\operatorname{ATRA}(+)$ differentiated cells using semi-quantitative
RT-PCR (Fig. 3f, g). All of these marker genes were not expressed in the differentiated cells.

These data indicated that ATRA had a positive effect on the process of differentiation into esophageal epithelium.

\section{Effects of ATRA on differentiation from FG to dAFG and from dAFG to EECs}

The effects of ATRA on organ specification from FG have been controversial $[8,20]$ (S-Fig. 5; also, see the "discussion" section). To clarify whether or not ATRA promotes differentiation of FG into dAFG in our protocol, we compared the expression of the dAFG marker genes SOX2 and p63 on Day 13 in the presence and absence of ATRA using quantitative RT-PCR (S-Fig. 6a). Our findings showed that ATRA significantly enhanced the expression of these marker genes as well as FABP5, a known target gene of ATRA (S-Fig. 6b).

We next assessed the effects of ATRA on the differentiation between dAFG and EEC (S-Fig. 6c), as no previous report has clarified the effect of ATRA treatment at this step (S-Fig. 5b, 5c). The results showed that, during transition from dAFG to EEC as well as from FG to dAFG, ATRA enhanced the expression of the EEC marker genes FABP5 and CK13 at Day 24 in the protocol (S-Fig. 6d).

\section{Effects of ATRA on differentiation into EECs via $\operatorname{RAR} \gamma$}

ATRA upregulates the downstream genes through RAR and RXR heterodimers. Of the three subtypes of RARs, RAR $\alpha$ and RAR $\beta$ are widely expressed in many tissues, while the expression of RAR $\gamma$ is limited but particularly strong in the skin and esophagus (S-Fig. 7a) [18].

To identify which subtype of RARs is important for esophageal differentiation, we used the small molecules AM580 (RAR $\alpha$-specific agonist), CD2314 (RAR $\beta$-specific agonist) and BMS961 (RAR $\gamma$-specific agonist) instead of ATRA (Fig. 4a and S-Fig. 7b) from Day 6 (FG) to Day 21 (EEC). RT-PCR showed that AM580 and CD2314 treatment resulted in a non-significant increasing trend in the expression of FABP5, which is a retinoid target gene [28] and whose expression is highest in the esophagus among human organ tissues [18], and SOX2, a general FG marker, but not the EEC-specific markers CK13, PAX9 and S100A14 (Fig. 4b and S-Fig. 7b). In contrast, BMS961 treatment significantly enhanced the expression of FABP5, CK13 and S100A14, similarly to ATRA treatment (Fig. 4c).

Therefore, in order to confirm whether or not ATRA enhances the differentiation of human esophagus via $\operatorname{RAR} \gamma$, we compared the expression of the esophageal markers in the resultant cells in the presence or absence of 
the RAR $\gamma$ antagonist LY2955303 during differentiation culture with ATRA (Fig. 4d). As shown in the Fig. 4e, RAR $\gamma$ antagonist treatment cancelled the enhancing effects of ATRA on the expression of esophageal-related genes, such as FABP5, SOX2, CK13, PAX9 and S100A14. Furthermore, to exclude the possibility that some off-target effect of LY2955303 contributed to the results of the experiments, we also used another RAR $\gamma$ antagonist, MM11253, and obtained the same results (S-Fig. 7c). In addition, we found that $\operatorname{RAR} \gamma$ was expressed in the FG (Day 6), and the expression increased as the differentiation progressed (Fig. 4f). These results indicate that the enhancing effect of ATRA on esophageal differentiation depends on $\operatorname{RAR} \gamma$ activation.

In addition, to address whether or not the increased SOX 2 expression induced by $\operatorname{RAR} \alpha$ and $\operatorname{RAR} \beta$ agonist (Fig. 4b and S-Fig. 7b) meant that these agonists induced differentiation into other tissues expressing SOX2, such as stomach and neuronal cells [18], we examined the expression of marker genes of stomach (TFF1, TFF2 and MUC5AC) and neuronal cells (SOX1 and PAX6) in the cells treated with $\operatorname{RAR} \alpha$ and $\operatorname{RAR} \beta$ agonist by semiquantitative RT-PCR. None of these marker genes was expressed in the cells (S-Fig. 8a and b), indicating that neither $\operatorname{RAR} \alpha$ nor $\operatorname{RAR} \beta$ agonist induced differentiation into these kinds of cells.

\section{Treatment with an $\operatorname{RAR} \gamma$-specific agonist instead of ATRA to induce stratified layers of cells expressing esophageal epithelial markers}

Finally, we examined whether or not treatment with an RAR $\gamma$ agonist instead of ATRA could also induce cells that recapitulated the expression of esophageal differentiation marker proteins and stratified squamous epithelial structures (Fig. 5a). After culture with the RAR $\gamma$-specific agonist using ALI, immunohistological analyses showed stratified cells with the obvious expression of SOX2, p63, CK5, E-cadherin, CK13, PAX9, Claudin-4 and Involucrin (Fig. 5b and S-Fig. 9).

\section{Discussion}

We successfully generated stratified cells expressing esophageal epithelial markers from hiPSCs with our original method and evaluated the significance of RA signaling on EEC induction after FG differentiation. RA signaling is known to be involved in the development of various tissues, such as brain, limbs, eye, lung and pancreas [29, 30]. However, the effect of RA signaling on the esophageal development remains to be clarified.
Several previous studies using mouse models [13, 14], but none using human cells, have reported that ATRA enhanced the terminal differentiation and proliferation of esophageal progenitor cells. However, these mouse studies did not address the role of RA signaling in the early steps of esophageal development. There have been only two previous papers on hiPSCs-derived esophageal differentiation. One of them did not use any RA signaling agonists at all in their protocol. The other (Triso et al. [8]) evaluated the effect of ATRA before (but not after) FG differentiation. The present study demonstrated that ATRA promotes both progression from FG to $\mathrm{dAFG}$, which is an esophageal progenitor (S-Fig. 6b), and from dAFG to esophageal epithelial differentiation (S-Fig. 6d) using hiPSC-derived models. Thus, to our knowledge, this is the first ever report describing the continuous effects of RA signaling on the processes of human EEC differentiation from FG.

In the present study, we demonstrated the enhancing effect of continuous activation of RA signaling on EEC induction after FG differentiation. In previous reports, the effect of RA signaling on the induction of the derivatives of FG has been controversial. We drew a comparative diagram (S-Fig. 5) showing our current protocol (a), the previous protocol for differentiation into posterior FG (PFG) (b) and ventral anterior FG (vAFG) (c) from foregut. Triso et al. [8] reported that ATRA treatment for 1 day during FG induction from DE at Day 5 in their protocol resulted in the increased expression of p63 and GATA4 at Day 9. p63 is expressed in not only dAFG but vAFG as well, which is a progenitor of lung cells [20, 31]. GATA4 is a marker of posterior FG (PFG) $[8,18,32]$ but not AFG. The authors also reported that ATRA treatment for four days after Day 5 promoted posterior FG differentiation rather than AFG [8] (S-Fig 5b). Thus, which fate specification of FG into dAFG, vAFG and PFG was promoted by ATRA has been unclear. Green et al. [20] showed that six days' treatment of FG with ATRA and CKFBE upregulated NKX2.1, a lung marker (S-Fig 5c). However, they did not assess the expression of the AFG marker SOX2, so whether ATRA treatment promotes $\mathrm{VAFG}$-specific differentiation or nonspecific AFG differentiation from FG has been unclear. In the present study, we demonstrated that treatment of FG with ATRA from Day 6 to Day 13 resulted in the enhanced expression of SOX2 and p63 (S-Fig. 6b), with no expression of NKX2.1 (S-Fig. 2a) or GATA4 (S-Fig. 2b). Furthermore, in our protocol, continuous treatment of FG with ATRA and CKFBE for 15-18 days enhanced neither lung markers, such as NKX2.1, SFTPB and SFTPC (Fig. 3f), nor PFG-derived stomach markers, such as TFF1, TFF2 and MUC5AC (Fig. 3g). These data indicated that ATRA promotes dAFG specification from FG. In addition, while Triso et al. [8] suggested that ATRA should be added prior to Day 6 (FG), we achieved efficient FG differentiation 
without ATRA by optimizing the concentration of CHIR during differentiation into DE (S-Fig. 1c and d). Differences in the identity of cells receiving ATRA among these reports might have led to these inconsistent outcomes of ATRA treatment.

No previous studies arguing the importance of retinoic acid in esophageal epithelial differentiation clarified which subtype of RA receptors played significant roles in the effect of RA. We identified for the first time RAR $\gamma$ as the RAR subtype whose activation promotes human EEC differentiation. This is compatible with the fact that RAR $\gamma$ is the predominant subtype of RAR in esophagus [18]. Regarding the differentiation of skin, which highly express RAR $\gamma$ (S-Fig. 3a), previous studies using mouse models reported that RA signaling via $\operatorname{RAR} \gamma$ played an important role $[28,33]$. In the present study, $\operatorname{RAR} \alpha$ and $\operatorname{RAR} \beta$ agonist treatment resulted in an increased expression of SOX2 without statistical significance, while no marked differences were noted in other esophageal markers (Fig. 4b and S-Fig. 7b). SOX2 is an early marker in the process of differentiation toward esophagus [21], and its expression is reduced in mature esophageal cells. RAR $\alpha$ and RAR $\beta$ agonist might promote the early phase of this process but not the maturation of esophageal cells. Notably, the combination of ATRA and RAR $\gamma$ antagonist did not increase the SOX2 expression, whereas that of RAR $\alpha$ and RAR $\beta$ agonist did. A previous study reported that ATRA binds to three types of RAR with different affinities [34]. ATRA may, therefore, have activated neither RAR $\alpha$ nor RAR $\beta$ in the current experiments, while the agonists AM580 and CD2314 activated RAR $\alpha$ and RAR $\beta$, respectively, and enhanced the expression of SOX2.

Of note, several reports argued that RA signaling had a suppressive effect on esophageal cancer [35] as well as skin cancer [36]. In addition, the decreased expression of DSG3 [37], SPRR3 [38] and MAL [39], which were all also decreased under conditions of ATRA absence in our experiment (Fig. 3e), were reported to be related to a poor prognosis of esophageal cancer. However, no report has clarified the relationship between the prognosis and expression or mutation of RAR $\gamma$ in esophageal cancer. In this context, we might need to focus on other hormones affecting esophageal differentiation as well. BMP4 and EGF were reported to promote esophageal differentiation from iPSCs [7] and proliferation of esophageal keratinocytes [40], respectively, so in the final step of our differentiation protocol, we employed BMP4 and EGF as well as ATRA and omitted the reagents (CHIR + FGF10 + KGF) reported to be required for inducing differentiation into the lung [20] in order to achieve specified differentiation to the esophagus. Future studies focusing on $\mathrm{RAR} \gamma$ signaling as well as these other relevant signals may provide some insight into not only esophageal differentiation but also the pathogenesis of esophageal cancer.

Acknowledgements We thank all of the members of the laboratory for their scientific comments and valuable discussion, as well as all of the staff. We also thank Yoko Matsuoka and Yukari Takatani for the administrative support.

This work was supported by a grant for Research Center Network for Realization of Regenerative Medicine (16817073, 19203662, 17935556) from the Japan Agency Medical Research and Development, AMED and JSPS KAKENHI (18958188, 16673738, 19K17490).

Open Access This article is licensed under a Creative Commons Attribution 4.0 International License, which permits use, sharing, adaptation, distribution and reproduction in any medium or format, as long as you give appropriate credit to the original author(s) and the source, provide a link to the Creative Commons licence, and indicate if changes were made. The images or other third party material in this article are included in the article's Creative Commons licence, unless indicated otherwise in a credit line to the material. If material is not included in the article's Creative Commons licence and your intended use is not permitted by statutory regulation or exceeds the permitted use, you will need to obtain permission directly from the copyright holder. To view a copy of this licence, visit http://creativecommons. org/licenses/by/4.0/.

\section{References}

1. Rodriguez P, Da Silva S, Oxburgh L, et al. BMP signaling in the development of the mouse esophagus and forestomach. Development. 2010;137:4171-6.

2. Romano RA, Smalley K, Magraw C, et al. DeltaNp63 knockout mice reveal its indispensable role as a master regulator of epithelial development and differentiation. Development. 2012;139:772-82.

3. Murry CE, Keller G. Differentiation of embryonic stem cells to clinically relevant populations: lessons from embryonic development. Cell. 2008;132:661-80.

4. Kajiwara M, Aoi T, Okita K, et al. Donor-dependent variations in hepatic differentiation from human-induced pluripotent stem cells. Proc Natl Acad Sci USA. 2012;109:12538-43.

5. Gotoh S, Ito I, Nagasaki T, et al. Generation of alveolar epithelial spheroids via isolated progenitor cells from human pluripotent stem cells. Stem Cell Rep. 2014;3:394-403.

6. McCracken KW, Cata EM, Crawford CM, et al. Modelling human development and disease in pluripotent stem-cell-derived gastric organoids. Nature. 2014;516:400-4.

7. Zhang Y, Yang Y, Jiang M, et al. 3D modeling of esophageal development using human PSC-derived basal progenitors reveals a critical role for notch signaling. Cell Stem Cell. 2018;23(516-29):e5.

8. Trisno SL, Philo KED, McCracken KW, et al. Esophageal organoids from human pluripotent stem cells delineate sox2 functions during esophageal specification. Cell Stem Cell. 2018;23(501-15):e7.

9. Osborn SL, Thangappan R, Luria A, et al. Induction of human embryonic and induced pluripotent stem cells into urothelium. Stem Cells Transl Med. 2014;3:610-9.

10. Pagliuca FW, Millman JR, Gurtler M, et al. Generation of functional human pancreatic beta cells in vitro. Cell. 2014;159:428-39. 
11. Petrova A, Celli A, Jacquet L, et al. 3D In vitro model of a functional epidermal permeability barrier from human embryonic stem cells and induced pluripotent stem cells. Stem Cell Reports. 2014;2:675-89.

12. Gouveia RM, Connon CJ. The effects of retinoic acid on human corneal stromal keratocytes cultured in vitro under serum-free conditions. Investig Opthalmol Visual Sci. 2013;54:7483-91.

13. DeWard AD, Cramer J, Lagasse E. Cellular heterogeneity in the mouse esophagus implicates the presence of a nonquiescent epithelial stem cell population. Cell Rep. 2014;9:701-11.

14. Doupe DP, Alcolea MP, Roshan A, et al. A single progenitor population switches behavior to maintain and repair esophageal epithelium. Science. 2012;337:1091-3.

15. Suzuki K, Koyanagi-Aoi M, Uehara K, et al. Directed differentiation of human induced pluripotent stem cells into mature stratified bladder urothelium. Scie Rep. 2019;9:10506.

16. Miyazaki T, Isobe T, Nakatsuji N, et al. Efficient adhesion culture of human pluripotent stem cells using laminin fragments in an uncoated manner. Sci Rep. 2017;7:41165.

17. Wang H, He L, Liu B, et al. Establishment and comparison of airliquid interface culture systems for primary and immortalized swine tracheal epithelial cells. BMC Cell Biol. 2018;19:10.

18. Fagerberg L, Hallstrom BM, Oksvold P, et al. Analysis of the human tissue-specific expression by genome-wide integration of transcriptomics and antibody-based proteomics. Mol Cell Proteomics. 2014;13:397-406.

19. Matsuno K, Mae SI, Okada C, et al. Redefining definitive endoderm subtypes by robust induction of human induced pluripotent stem cells. Differentiation. 2016;92:281-90.

20. Green MD, Chen A, Nostro MC, et al. Generation of anterior foregut endoderm from human embryonic and induced pluripotent stem cells. Nat Biotechnol. 2011;29:267-72.

21. Zhang Y, Jiang M, Kim E, et al. Development and stem cells of the esophagus. Semin Cell Dev Biol. 2017;66:25-35.

22. Que J, Okubo T, Goldenring JR, et al. Multiple dose-dependent roles for Sox 2 in the patterning and differentiation of anterior foregut endoderm. Development. 2007;134:2521-31.

23. Kechele DO, Wells JM. Recent advances in deriving human endodermal tissues from pluripotent stem cells. Curr Opin Cell Biol. 2019;61:92-100.

24. Daniely Y, Liao G, Dixon D, et al. Critical role of p63 in the development of a normal esophageal and tracheobronchial epithelium. Am J Physiol Cell Physiol. 2004;287:C171C181181.

25. Wong AP, Bear CE, Chin S, et al. Directed differentiation of human pluripotent stem cells into mature airway epithelia expressing functional CFTR protein. Nat Biotechnol. 2012;30:876-82.

26. Wilson HK, Canfield SG, Hjortness MK, et al. Exploring the effects of cell seeding density on the differentiation of human pluripotent stem cells to brain microvascular endothelial cells. Fluids Barriers CNS. 2015;12:13.
27. Sherrill JD, Kc K, Wu D, et al. Desmoglein-1 regulates esophageal epithelial barrier function and immune responses in eosinophilic esophagitis. Mucosal Immunol. 2014;7:718-29.

28. Gericke J, Ittensohn J, Mihaly J, et al. Regulation of retinoidmediated signaling involved in skin homeostasis by RAR and RXR agonists/antagonists in mouse skin. PLoS ONE. 2013;8:e62643.

29. Rhinn M, Dolle P. Retinoic acid signalling during development. Development. 2012;139:843-58.

30. Duester G. Retinoic acid synthesis and signaling during early organogenesis. Cell. 2008;134:921-31.

31. Morrisey EE, Rustgi AK. The lung and esophagus: developmental and regenerative overlap. Trends Cell Biol. 2018;28:738-48.

32. Rojas A, Schachterle W, Xu SM, et al. Direct transcriptional regulation of Gata4 during early endoderm specification is controlled by FoxA2 binding to an intronic enhancer. Dev Biol. 2010;346:346-55.

33. Chapellier B, Mark M, Messaddeq N, et al. Physiological and retinoid-induced proliferations of epidermis basal keratinocytes are differently controlled. Embo J. 2002;21:3402-13.

34. Idres N, Marill J, Flexor MA, et al. Activation of retinoic acid receptor-dependent transcription by all-trans-retinoic acid metabolites and isomers. J Biol Chem. 2002;277:31491-8.

35. Li N, Lu Y, Li D, et al. All-trans retinoic acid suppresses the angiopoietin-Tie2 pathway and inhibits angiogenesis and metastasis in esophageal squamous cell carcinoma. PLoS ONE. 2017; 12:e0174555.

36. Cheepala SB, Yin W, Syed Z, et al. Identification of the B-Raf/ Mek/Erk MAP kinase pathway as a target for all-trans retinoic acid during skin cancer promotion. Mol Cancer. 2009;8:27.

37. Fang WK, Gu W, Liao LD, et al. Prognostic significance of desmoglein 2 and desmoglein 3 in esophageal squamous cell carcinoma. Asian Pac J Cancer Prev APJCP. 2014;15:871-6.

38. Zhang Y, Feng YB, Shen XM, et al. Exogenous expression of Esophagin/SPRR3 attenuates the tumorigenicity of esophageal squamous cell carcinoma cells via promoting apoptosis. Int $\mathbf{J}$ Cancer. 2008;122:260-6.

39. Mimori K, Shiraishi T, Mashino K, et al. MAL gene expression in esophageal cancer suppresses motility, invasion and tumorigenicity and enhances apoptosis through the Fas pathway. Oncogene. 2003;22:3463-71.

40. Qureshi FG, Duncan MD, Harmon JW, et al. EGF and IGF-1 synergistically stimulate proliferation of human esophageal epithelial cells. J Surg Res. 1999;69:354-8.

Publisher's Note Springer Nature remains neutral with regard to jurisdictional claims in published maps and institutional affiliations. 